infrastructure, including standardised training, and more professionals to sustain and expand the model. Another approach could be to train available medical officers to take up the role of the mental health professional in resource-poor rural areas like those covered by the project. The Shifa approach requires devoted families living within supportive villages, which may not always be present, particularly in war-torn areas. The data collected within the project are based on nonstandard measures and rating techniques that need validation. Finally, the emphasis on helping people with the most severe disorders - which is necessary due to the scarce resources - inevitably results in less attention to others who also need help.

\section{Conclusions}

Project Shifa demonstrates a cost-effective approach to psychiatric care and rehabilitation in rural India. Other healthcare teams in India and in other low- and middle- income countries, which face similar challenges, might find some of the strategies employed by the project useful. By relying on a lay workforce of health workers and families, using low-cost medications and a home-based approach, the project has demonstrated that psychiatric outreach, treatment and rehabilitation can be effectively implemented at very low cost in a sizeable rural area with only a single available trained mental health professional.

\section{References}

Becker A. E. \& Kleinman A. (2013) Mental health and the global agenda. New England Journal of Medicine, 369, 66-73.

Chatterjee S., Patel V., Chatterjee A., et al (2003) Evaluation of a community based rehabilitation model for chronic schizophrenia in a rural region of India. British Journal of Psychiatry, 182, 57-62.

Collins P. Y., Patel V., Joestl S. S., et al (2011) Grand challenges in global mental health. Nature, 475, 27-30.

Corrigan P. W. (2016) Principles and Practice of Psychiatric Rehabilitation: An Empirical Approach, 2nd edn. Guilford Press.

Drake R. E., Bajraktari I. \& Tansella M. (2014a) Technology and behavioral health: an implementation challenge. Epidemiology and Psychiatric Sciences, 23, 313-315.

Drake R. E., Binagwaho A., Castillo Martel H., et al (2014b) Mental healthcare in low and middle income countries should not replicate the inefficient, inaccessible, ineffective Western model. BMJ, 349, g7086.

Kumar A. (2011) Mental health services in India: challenges and prospects. Health, 3, 757-761.

Mulley A. G., Richards T. \& Abbassi K. (2015) Delivering health with integrity of purpose: Health systems must learn how to co-produce and deliver services that patients and the public value. BMJ, 351 h4448.

National Institute of Health and Family Welfare (2010) National Mental Health Program. http://www.nihfw.org/NationalHealth Programme/NATIONALMENTALHEALTHPROGRAMME.html (accessed 1 December 2017).

WHO (2011) Mental Health Atlas: Country Profiles on Mental Health Resources. World Health Organization.

WHO (2013) Mental Health Action Plan. World Health Organization.

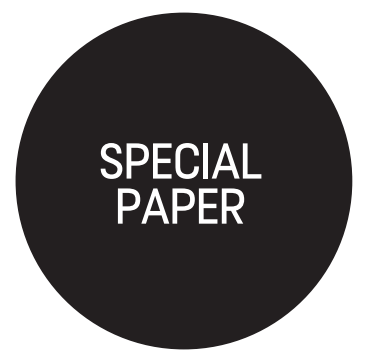

\title{
Nothing about us without us: the importance of local collaboration and engagement in the global study of autism
}

\author{
Rosa A. Hoekstra, ${ }^{1}$ Fikirte Girma, ${ }^{2}$ Bethlehem Tekola ${ }^{3}$ and Zemi Yenus ${ }^{4}$
}

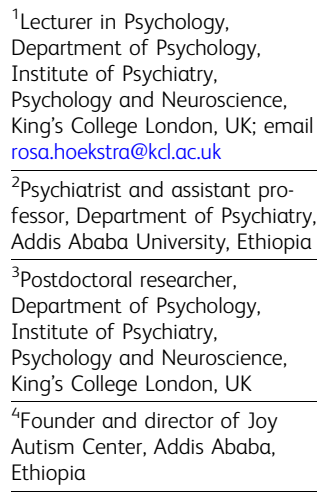

Our current understanding of autism and other developmental disorders is primarily based on research conducted in high-income countries, predominantly in North America and Europe. Even within high-income countries, White participants are overrepresented in autism research. There is now increased recognition that a more global and diverse research representation is warranted. This paper argues that in order for global and diverse research efforts to be effective, it is essential to collaborate and engage with local experts and stakeholders, including local researchers, clinicians and representatives from governmental and non-governmental organisations. Such collaborations ensure that studies use culturally appropriate methods and materials, and that research findings are interpreted taking local context into account. Ultimately, these collaborations build local capacity and foster the development of culturally and contextually appropriate interventions that address locally perceived needs. The adage 
Acknowledgements. This research would not have been possible without the valuable contributions of Dr Abebaw Fekadu, Awoke Mihretu, Dr Charlotte Hanlon, Dejene Tilahun, Prof. Markos Tesfaye, Wubalem Adamu, Dr Yonas Baheretibeb and our various stakeholder, especially the members of the PST adaptation advisory board.

Conflicts of interest. None.

doi:10.1192/bji.2017.26

(C) The Authors 2018. This is an Open Access article, distributed under the terms of the Creative Commons Attribution-

NonCommerical-NoDerivatives commons. org/licenses/by-nc-nd/4.0/), which permits non-commercial re-use, distribution, and reproduction in any medium, provided the original work is unaltered and is properly cited. The written permission of Cambridge University mission of Cambridge University
Press must be obtained for commercial re-use or in order to create a derivative work. 'nothing about us without us' is vital to global autism research.

Mental health problems and developmental disorders affect 10 to $20 \%$ of children globally (Kieling et al, 2011). Although 90\% of all children live in low- and middle-income countries (LMIC), most child mental health research is conducted in high-income countries (HIC) (Kieling et al, 2011), and fewer than 7\% of developmental psychology studies are from nonWestern countries (Nielsen et al, 2017). Although the gap in global mental health research was highlighted more than 15 years ago (Patel \& Sumathipala, 2001), its relevance and importance to the study of child development and autism has only recently started to receive attention (Durkin et al, 2015; Nielsen et al, 2017). Most autism research is conducted in North America and Europe, with only a small proportion of studies coming from LMIC. This research divide has two important drawbacks (Durkin et al, 2015). First, our knowledge of autism and its causes may be incomplete and biased. Second, because research is often linked to capacity building, the lack of research in LMIC is likely to contribute to a growing inequity in access to evidence-based services and support.

There is currently a real impetus for global research on autism and other developmental disorders. The journal Autism recently published a special issue on Global Autism Research, and several funding mechanisms, including the Global Alliance for Chronic Diseases and the UK's Global Challenges Research Fund, explicitly welcome applications related to mental health and developmental disorders in LMIC. To effectively capitalise on the increased attention paid to global autism research, this paper argues for HIC researchers to collaborate with local partners. Over the past 5 years, our team - based in Ethiopia and the UK - has conducted a set of studies on autism and other developmental disorders in Ethiopia. The key lesson we learned was the importance of engagement with local researchers and a wider group of stakeholders, including clinicians and representatives from governmental and non-governmental organisations (NGOs). We present examples from our experiences to illustrate how, without this local collaboration, our efforts could have been counterproductive, potentially resulting in research findings that were biased or in interventions that were ineffective or even harmful.

\section{The case for using local expertise}

First, working with a team of local researchers and stakeholders ensures the use of culturally appropriate research methods. Data collection methods common in HIC, such as paper-and-pen questionnaires or online surveys, are less suitable for use in LMIC such as Ethiopia, owing to low literacy rates and a strong oral tradition. Previous research led by Ethiopian colleagues (e.g. Shibre et al, 2006) indicated that completion of a questionnaire through a face-to-face interview with a lay interviewer is a culturally appropriate alternative. The local researchers in our team guided the design of these study aspects, including appropriate training for the lay interviewers (Tilahun et al, 2017a, b).

Similarly, local expert input is needed to ensure that questionnaires fit local cultural norms and context. For example, we found that the anchoring of items of a caregiver involvement measure, originally developed in the USA, required cultural adaptation. Whereas enthusiasm tends to be displayed openly in the USA, equivalent levels of involvement will typically be shown in less overt terms in Ethiopia. Thus, the anchors providing examples of what typifies a 'very enthusiastic' or a 'not enthusiastic' caregiver required adjustment.

Local perspectives may also prove essential when interpreting research findings. Our survey in caregivers of children with developmental disorders indicated that the practice of restraining a child is relatively common (Tilahun et al, 2016). Subsequent discussion of this finding during a stakeholder workshop taught us that restraint is not typically used as punishment, but a last-resort measure to protect the child from harm when there is no one to look after the child (Tekola et al, 2016). Thus, these results should not be interpreted in terms of abuse, but rather in the light of lack of available support. Consequently, efforts to reduce restraining of children with developmental disorders are more likely to be successful if they focus on improving support and care systems for families, rather than an education intervention solely conveying the message that restraining is harmful.

Local input is also essential when designing interventions. When we developed the mental health module of an education programme for Ethiopian community health workers (Tilahun et al, 2017b), local experts indicated that we should not emphasise the strong genetic influences on conditions such as schizophrenia and autism. An emphasis on high heritability could have had the unintended consequence of replacing existing stigmatising beliefs in the community with stigma associated with a 'genetic taint'.

Perhaps most importantly, local collaboration and engagement are essential to ensure that the intervention addresses a locally perceived need, suits the country's health and education system, and is supported by local stakeholders. For an intervention to be sustainable and have scope for scale-up, the programme needs to be 'owned' by local partners. In an ongoing project, we are adapting and evaluating a parent skills training (PST) programme, developed by the World Health Organization, for use in Ethiopia. We set 
up a PST adaptation advisory board, consisting of local experts and stakeholders, who over the past 2 years have provided invaluable feedback on the draft content and delivery of the programme. Without local input, the training could have inadvertently set wrong expectations (e.g. that the intervention may result in a cure; Tilahun et al, 2016), glossed over pertinent local issues or used culturally inappropriate intervention strategies.

Finally, working with local universities contributes to capacity building for research in the country. Although global research has burgeoned, recent analyses (Kelaher et al, 2016; Nielsen et al, 2017) suggest that the scientific articles reporting on these studies are primarily written by researchers from HIC. HIC researchers should invest in collaborating with local researchers and the training of local students, making sure they get the opportunity to write papers and submit to international peer-reviewed journals. These researchers and students are likely to take up prestigious posts and be involved in training future cohorts of local experts, thereby allowing for future expansion in research and mental health services.

\section{How to achieve local collaboration and engagement}

In many LMIC, in the absence of comprehensive governmental services, grassroots NGOs have been founded to address pertinent needs. The last author of this paper (Z.Y.) established Ethiopia's first autism school; following her pioneering work, additional schools for children with developmental disorders have opened (Tekola et al, 2016). A useful way to initiate a local network is through these local organisations, because they will have wide-ranging practical experience and knowledge of the challenges faced by local families. Connections with researchers can be built through local universities. Local researchers do not (yet) have to be autism experts; a public health or mental health researcher will still be well qualified to give input in locally appropriate research methods and study designs. It is this mix of partners that is important: local stakeholders are experts in the perceived needs and practical considerations, but are less well placed to comment on research techniques; in turn, researchers may miss subtle issues with research instruments if they are not also scrutinised by stakeholders with first-hand experience with autism.

The optimal method for local engagement will depend on the culture and context. Low literacy rates and poor internet access make written consultations less suitable for use in Ethiopia. In our experience, face-to-face workshop-style stakeholder consultations work best (Tekola et al, 2016). Once a network is established, it is important to sustain it by keeping stakeholders involved in each step of the research process, from the study design phase, through regular progress updates, to presentation of preliminary findings and dissemination of the final research report.

\section{Global benefits of local collaboration and engagement}

Although the preceding paragraphs illustrate our experiences in one particular low-income country, these are likely to have relevance more widely. High levels of stigma (Divan et al, 2012) and lack of culturally appropriate screening and diagnostic tools (Durkin et al, 2015) and interventions are prominent issues in most LMIC. Research in LMIC might also inform strategies to improve access to services and research in hard-to-reach groups within HIC. Ethnic minorities are underrepresented in research in HIC (West et al, 2016); autism tends to be underdiagnosed and diagnosed late in children of ethnic minorities, and parents of these children report receiving lowerquality healthcare (Magaña et al, 2015). Research findings from LMIC might thus provide clues to how to better reach these under-served groups in HIC.

In fact, the call for inclusion of local partners in research equally applies to research in hardto-reach groups within HIC. Researchers and students from ethnic minorities are underrepresented at research-intensive universities. The inclusion of such groups in research teams is more likely to lead to innovative research designs that will promote successful recruitment and retention of diverse participants in research studies.

\section{Conclusion}

For research in under-served communities to be successful (whether in LMIC or in subgroups in HIC), researchers at elite universities in HIC need to make serious efforts to engage with academics and stakeholders that reflect the community they wish to study. Only through this 'nothing about us without us' approach can we ensure that our research uses culturally appropriate methods and materials, and that findings are interpreted according to local cultural context and result in sustainable interventions that address locally perceived needs.

\section{Funding}

The research referred to in this paper was financially supported by grants from Autism Speaks (\#7770 and \#9817), the Medical Research Council (UK; \#MR/P020844/1) and a Slifka/Ritvo award funded by the Alan B. Slifka foundation.

\section{References}

Divan G., Vajaratkar V., Desai M. U., et al (2012) Challenges, coping strategies, and unmet needs of families with a child with autism spectrum disorder in Goa, India. Autism Research, 5, 190-200.

Durkin M.S., Elsabbagh M., Barbaro J., et al (2015) Autism screening and diagnosis in low resource settings: challenges and opportunities to enhance research and services worldwide. Autism Research, 8, 473-476.

Kelaher M., Ng L., Knight K., et al (2016) Equity in global health research in the new millennium: trends in first-authorship for randomized controlled trials among low- and middle-income country researchers 1990-2013. International Journal of Epidemiology, 45, 2174-2183. 
Kieling C., Baker-Henningham H., Belfer M., et al (2011) Global mental health 2. Child and adolescent mental health worldwide: evidence for action. Lancet, 378, 1515-1525.

Magaña S., Parish S. L. \& Son E. (2015) Have racial and ethnic disparities in the quality of health care relationships changed for children with developmental disabilities and ASD? American Journal on Intellectual and Developmental Disabilities, 120, 504-513.

Nielsen M., Haun D., Kärtner J., et al (2017) The persistent sampling bias in developmental psychology: a call to action. Journal of Experimental Child Psychology, 162, 31-38.

Patel V. \& Sumathipala A. (2001) International representation in psychiatric literature: survey of six leading journals. British Journal of Psychiatry, 178, 406-409.

Shibre T., Alem A., Tekle-Haimanot R., et al (2006) Perception of stigma in people with epilepsy and their relatives in Butajira, Ethiopia. Ethiopian Journal of Health Development, 20, 170-176.

Tekola B., Baheretibeb Y., Roth I., et al (2016) Challenges and opportunities to improve autism services in low-income countries: lessons from a situational analysis in Ethiopia. Global Mental Health, 3, e21.

Tilahun D., Hanlon C., Fekadu A., et al (2016) Stigma, explanatory models and unmet needs of caregivers of children with developmental disorders in a low-income African country: a crosssectional facility-based survey. BMC Health Services Research, 16, 152.

Tilahun D., Hanlon C., Araya M., et al (2017a) Training needs and perspectives of community health workers in relation to integrating child mental health care into primary health care in a rural setting in sub-Saharan Africa: a mixed methods study. International Journal of Mental Health Systems, 11, 15.

Tilahun D., Fekadu A., Tekola B., et al (2017b) Ethiopian community health workers' beliefs and attitudes towards children with autism: impact of a brief training intervention. Autism, doi: 10.1177/ 1362361317730298

West E. A., Travers J. C., Kemper T. D., et al (2016) Racial and ethnic diversity of participants in research supporting evidence-based practices for learners with Autism spectrum disorder. The Journal of Special Education, 50, 151-163.

\title{
SPECIAL PAPER
}

\section{Mental health information systems in resource-challenged countries: experiences from India}

\author{
Shalini Ahuja, ${ }^{1}$ Rahul Shidhaye, ${ }^{2}$ Maya Semrau, ${ }^{3}$ Graham Thornicroft ${ }^{4}$ and \\ Mark Jordans ${ }^{3}$
}

\begin{abstract}
${ }^{1}$ King's College London, UK; email shalini.ahuja@kcl.ac.uk

${ }^{2}$ Public Health Foundation of India, India

${ }^{3}$ Institute of Psychiatry,

Psychology and Neurosciences,

King's College London, UK

${ }^{4}$ Community Mental Health,

Institute of Psychiatry,

Psychology and Neurosciences,

King's College London, UK

The views expressed are those of the author(s) and not necessarily those of the National Health

those of the National Health
Service, the National Institute for

Health Research or the

Department of Health.

\section{Declaration of interest. None}

\section{doi:10.1192/bji.2017.6}

(c) The Authors 2018. This is an Open Access article, distributed under the terms of the Creative Commons Attribution-

NonCommercial-NoDerivatives

NonCommercial-NoDerivatives
licence (http://creativecommons. org/licenses/by-nc-nd/4.0/), which permits non-commercial re-use, distribution, and reproduction in any medium, provided the original work is unaltered and is ginal work is unaltered and is properly cited. The written permission of Cambridge University Press must be obtained for commercial re-use or in order to create a derivative work.
\end{abstract}

\begin{abstract}
Mental health information systems are increasingly being used to measure the effectiveness of mental health interventions. Little or no data is available for mental health service availability and service uptake in lowand middle-income countries. Through a narrative review, this paper illustrates the importance of routine monitoring data and suggests methods for developing, implementing and evaluating mental health indicators in low- and middle-income countries with a primary focus on India.
\end{abstract}

Only $2 \%$ of people with mental disorders in lowand middle-income countries (LMICs) - where $85 \%$ of the world's population lives - receive treatment (Eaton et al, 2011). In India, one in ten people receive evidence-based interventions for such disorders (Charlson et al, 2016) and for every 100000 people in India there are 0.6 mental health professionals, delivering care through mental hospitals (43 in the country) and primary care settings (WHO, 2011). Less than $1 \%$ of the national healthcare budget is allocated to mental health in countries such as India and China (Patel et al, 2016). In India, even with a favourable policy environment and a national programme in place, there is poor service provision and therefore negligible data available for future planning, implementation and research (Shidhaye et al, 2015). In an effort to decrease the 'gap between the true prevalence of a disorder and the number of affected people who receive treatment - called the 'treatment gap' - the scaling up of mental health services has been advocated in LMICs. Scalability implies the capacity to expand a health intervention to a large scale without reducing its effectiveness. However, insufficient evaluation of how best to deliver services and poor availability of adequate information for decision making are hindering the scaling up of mental health services in LMICs (Eaton et al, 2011).

Mental health information systems (MHIS), used for measuring and managing mental health service delivery, have become increasingly important in improving the effectiveness of mental healthcare (Jordans et al, 2016). Health management information systems (HMIS), including those for mental health, ensure the collection, processing and reporting of data and are specifically designed to assist health management policy and planning (WHO, 2004).

These systems are described as the building blocks of a health system and they can consistently provide accurate information enabling planning and evaluation of mental health service delivery (WHO, 2004). Furthermore, when combined with 